\title{
The kinematically decoupled core of MCG-6-30-15
}

\author{
Sandra I. Raimundo \\ SISSA - International School for Advanced Studies, via Bonomea, 265, 34136 Trieste, Italy \\ email: sandra.raimundo@sissa.it
}

\begin{abstract}
The Seyfert galaxy MCG-6-30-15 has recently been observed in the infrared using SINFONI on the VLT, reaching a very high spatial resolution of 0.1 arcsec. This allowed us for the first time to detect a stellar kinematically decoupled core in the inner $\mathrm{r}<125 \mathrm{pc}$ of the galaxy. Here we summarise the main theories for the formation of the decoupled core and the implications on the study of this galaxy.
\end{abstract}

Keywords. galaxies:active, galaxies: Seyfert, galaxies: individual(MCG-6-30-15), accretion

\section{Introduction}

Stellar kinematically decoupled cores (KDCs) are characterised by the presence of a stellar core with kinematics and angular momentum distinct from the main body of the galaxy. KDCs, of which counter-rotating cores are a sub-group, can be defined by an abrupt change of more than 30 degrees in the local kinematic orientation of the galaxy (e.g. Krajnovic et al. 2011). It is unclear what mechanisms are responsible for the KDC formation, but the main theories involve the inflow of material (stars/gas) with a distinct angular momentum into the centre of the galaxy. This can be via 1) a dry merger with a small elliptical galaxy (Kormendy 1984); 2) a merger with a small gas rich object (Franx \& Illingworth 1988); 3) inflow of gas due to an early major merger between two gas rich galaxies (Hernquist \& Barnes 1991) or 4) inflow of gas due to secular processes that transport already counter-rotating gas from the outskirts to the centre of the galaxy. In the gas inflow case (cases 2), 3) and 4)), the stellar KDC would be the result of in-situ star formation after the decoupled gas settles in the galaxy core.

The object of the study presented here is the galaxy MCG-6-30-15, an active galaxy of the type S0 at $z=0.0077$. This galaxy recently became relevant to the discussion above due to the discovery of a small stellar kinematically decoupled core in its core. The details of its discovery and main implications will be described in the following sections.

\section{Results}

We recently discovered a stellar kinematically decoupled core in the active galaxy MCG-6-30-15. The decoupled core was observed using the first $H$-band infrared integral field observations of this source, carried out with SINFONI on the VLT. Its presence is noticeable in the inner $\mathrm{r}<125 \mathrm{pc}$ region of the galaxy, and can be distinguished due to a different rotation orientation of the stars (Fig. 1). The high spatial resolution of these data was the main reason why we were able to find this previously unknown decoupled core. One of the key methods of this work was the subtraction of the broad hydrogen Brackett AGN emission lines. These lines are very strong in the central pixels and dominate over the wavelength range of interest, making the stellar absorption features hard 


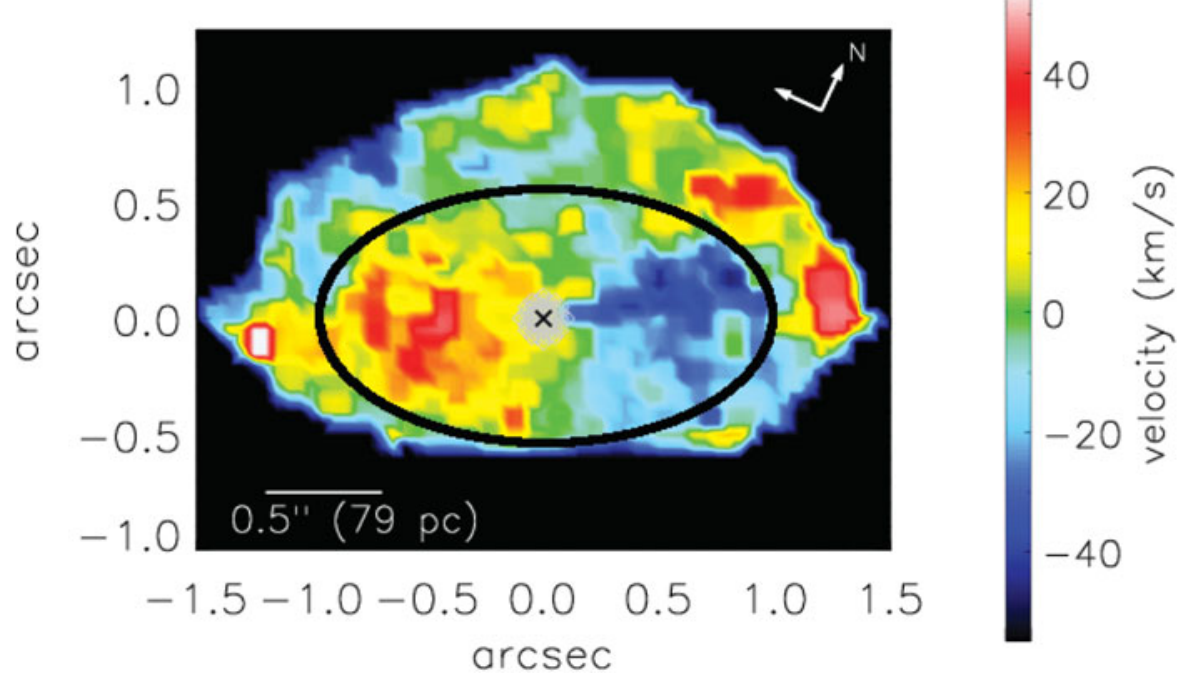

Figure 1. Two-Dimensional map of line of sight velocity from spectral fitting. The black cross indicates the AGN position. Positive and negative velocities correspond to movement away and towards the observer respectively. A kinematically decoupled core is observed in the velocity map and highlighted with the black ellipse. Within this region the stars rotate in the clockwise direction while in the outer regions they rotate in the opposite direction (seen as a change of velocity direction in the top left and top right regions of the map).

to identify. We adopted a method that can be used in future observations of bright AGN, to remove these broad emission lines and determine the kinematics from the underlying stellar absorption features (Raimundo et al. 2013). The stellar kinematics were mapped out to a radius of $\mathrm{r}<190 \mathrm{pc}(1.2$ arcsec $)$ and the gas dynamics, traced by the [Fe II] $1.64 \mu \mathrm{m}$ emission line, was mapped out to $\mathrm{r}=130 \mathrm{pc}(0.8 \operatorname{arcsec})$. Assuming that the [Fe II] was caused by supernova shocks we determined the age of the stellar population in the core to be $\sim 65 \mathrm{Myr}$. If in fact the supernova trace the kinematically decoupled stellar population, then one of the possible scenarios for the KDC formation would be gas inflow and subsequent star formation.

\section{Implications}

KDCs are relatively rare in S0 galaxies such as MCG-6-30-15 $(<10 \%$ Kuijken et al. 1996). Out of the more than 50 KDCs discovered and published in the literature, MCG-6$30-15$ is the one with the highest AGN X-ray luminosity $\left(\mathrm{L}_{\mathrm{X}(2-10) \mathrm{keV}}=4 \times 10^{42} \mathrm{erg} \mathrm{s}^{-1}\right)$. Most of the galaxies are quiescent and the ones with active nuclei have lower $\mathrm{X}$-ray luminosities typical of low-ionisation nuclear emission regions (LINERs). What is still unknown is what mechanism was responsible for the formation of the decoupled core. The fact that this galaxy shows a dust lane may indicate that there was a merger event in its past. Clues on its origin may come from the star formation history. It is expected that the stellar properties of the decoupled cores and the main body of the galaxy will be different. McDermid (2006) found that some of the KDCs in the SAURON earlytype galaxy sample consisted of younger stellar populations and these were typically the smallest KDCs in size (Fig. 16 of their paper). The larger KDCs had in general stellar populations that were indistinguishable from the rest of the galaxy. This could be 
related with a different formation mechanism, where the young KDCs are formed from gas inflow into the centre of the galaxy which then forms a younger stellar population, while the older KDCs were formed mainly from dry mergers at early times. As small KDCs can only be detected with high spatial resolution observations, we are probably severely underestimating the number of galaxies with KDCs. Since integral field spectroscopy observations are one of the best techniques to reach this goal, we are also likely biased to low luminosity AGN or quiescent galaxies, since strong AGN hosts are usually excluded from the list of targets due to AGN contamination in the emission from the galaxy. MCG-6-30-15 is therefore a unique and important case study since it has both a bright AGN and a small KDC. In the simplified scenario of McDermid (2006), due to the size of the KDC in MCG-6-30-15 and hints of a younger stellar population, we would expect the KDC to have been formed from gas inflow into the centre of the galaxy.

\section{How can we identify gas inflow or distinguish between formation scenarios?}

While it is very hard to determine the exact mechanism that caused the KDC formation, clues can be obtained from the observable properties of the gas and stars. Even if we cannot constrain the KDC formation mechanism, we can investigate if it is related with recent gas inflow. The ionised gas ([Fe II $]$ ) seems to have the same direction of rotation of the counter-rotating core, but due to the small field-of-view of our observations and it being possibly associated with supernova shocks or even shocks by AGN driven outflows, it is hard to tell if the gas is co-rotating or counter-rotating with the stars. The molecular gas, expected to trace the cooler gas and the reservoir for black hole fuelling will provide an unambiguous test for the gas rotation direction. If the molecular gas is rotating with the outer main part of the galaxy, then it was probably a result of gas inflow via secular processes after the KDC formation and is disconnected from it. If on the other hand the molecular gas shares the same KDC kinematics, then it is likely that both are the result of the same gas inflow event, and the hypothesis of a dry merger becomes very unlikely. New observations are planned for this year using SINFONI on the VLT, to map the molecular gas in the inner $\sim 600 \mathrm{pc}$ of the galaxy and determine the gas dynamics. Our planned observations will provide a test to the relation between the molecular gas and the decoupled stellar core. Another clue can come from a detailed study of the stellar population in the KDC and in the outer part of the galaxy. If the stellar population in the KDC is indistinguishable from the rest of the galaxy, then the KDC formation must have happened at early times and it will not be associated with recent gas inflow.

Why are we interested in recent gas inflow? The main reason is that this galaxy is active and the black hole is currently accreting from its surroundings. Inflow of gas during the decoupled core formation could provide a mechanism for AGN fuelling, although there is still the problem of transporting the gas from the decoupled core scales to the scale of the accretion disc. MCG-6-30-15 was famously the first galaxy to show a broad iron emission line in the X-ray spectrum which was used to constrain the black hole spin (Tanaka et al. 1995). The most recent measurements indicate a high spin of $a>0.98$ (Brenneman \& Reynolds 2006). This galaxy and its small decoupled core provide an interesting laboratory for tests on accretion physics. From theoretical models, the black hole spin increases due to continuous accretion of material with similar angular momentum. If the black hole has been accreting from counter-rotating gas for the past $65 \mathrm{Myr}$, it could mean that the spin has decreased in relation to its higher initial value. 
More details on the methods and results can be found in Raimundo et al. (2013).

\section{References}

Brenneman, L. W. \& Reynolds, C. S. 2006, ApJ, 652, 1028

Franx, M. \& Illingworth, G. D. 1988, ApJ, 327, 55

Hernquist, L. \& Barnes, J. 1991, Nature, 354, 210

Kormendy, J. 1984, ApJ, 287, 577

Krajnović, D., Emsellem, E., Cappellari, M., Alatalo, K., Blitz, L., Bois, M., Bournaud, F., Bureau, M., Davies, R. L., Davis, T. A., de Zeeuw, P. T., Khochfar, S., Kuntschner, H., Lablanche, P.-Y., McDermid, R. M., Morganti, R., Naab, T., Oosterloo, T., Sarzi, M., Scott, N., Serra, P., Weijmans, A.-M., \& Young, L. M. 2011, ApJ, 441, 2923

Kuijken, K. \& Fisher, D. Merrifield, M. R. 1996, MNRAS, 283, 543

McDermid, R. M., Emsellem, E., Shapiro, K. L., Bacon, R., Bureau, M., Cappellari, M., Davies, R. L., de Zeeuw, T., Falcón-Barroso, J., Krajnović, D., Kuntschner, H., Peletier, R. F., \& Sarzi, M. 2006, MNRAS, 373, 903

Raimundo, S. I., Davies, R. I., Gandhi, P., Fabian, A. C., Canning, R. E. A., \& Ivanov, V. D. 2013, MNRAS, 431, 2294

Tanaka, Y., Nandra, K., Fabian, A. C., Inoue, H., Otani, C., Dotani, T., Hayashida, K., Iwasawa, K., Kii, T., Kunieda, H., Makino, F., \& Matsuoka, M., 1995, Nature, 375, 659 